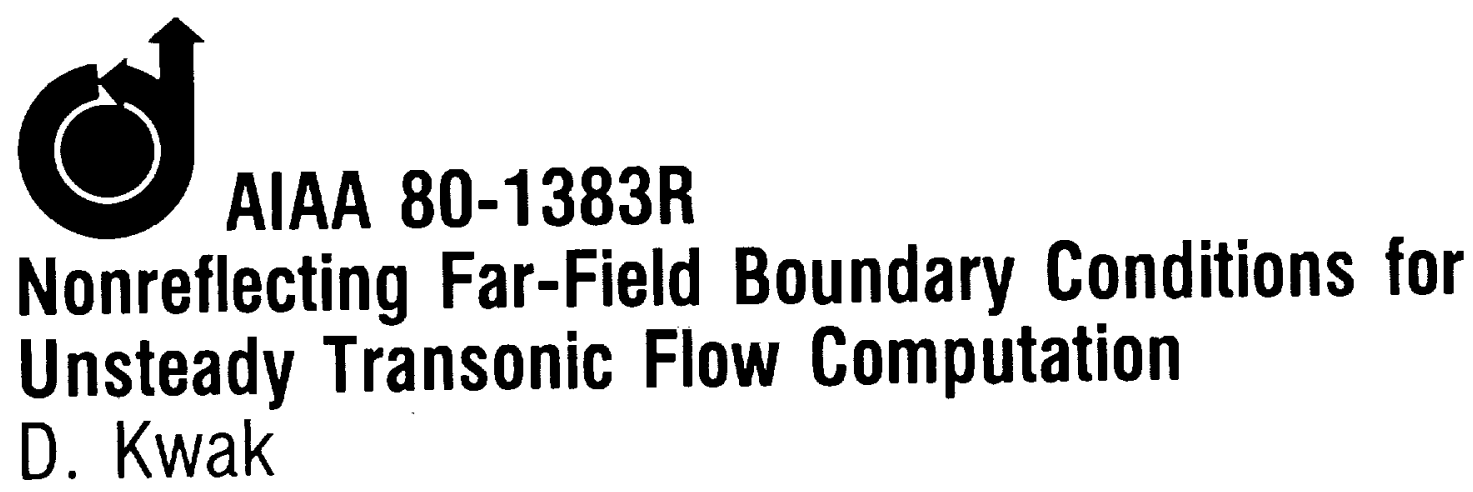




\title{
Nonreflecting Far-Field Boundary Conditions for Unsteady Transonic Flow Computation
}

\author{
D. Kwak* \\ NASA Ames Research Center, Moffett Field, Calif.
}

\begin{abstract}
The approximale nonreflecting far-field boundary condition, as proposed by Engquist and Majda, is implemented in the computer code LTRAN2. This code solves the implicit finile-difference representation of the small-disturbance equations for unsteady Iransonic flows about airfoils. The nonreflecting boundary condition and the description of the algorithm for implementing these conditions in LTRAN2 are discussed. Various cases are computed and compared with results from the older, more conventional procedures. One concludes that the nonreflecting far-field boundary approximation allows the far-field boundary to be located closer to the airfoil; this permits decrease in the computer time required to obtain the solution through the use of fewer mesh points.
\end{abstract}

\section{Introduction}

$\mathbf{T}$ $O$ compute time-dependent flows over an infinite region using finite-difference procedures requires either that the problem be reduced 10 the one in a finite domain via a coordinate transformation, or that boundaries be placed at a finite distance from the body and the boundary conditions modified along them. When the solution at infinity is simple, a coordinate transformation technique can be used. However, for many practical problems, especially when the flow is singular at infinity, the coordinate transformation method is not applicable. In such cases, computational simulations of unsteady flows in an unbounded region are performed on grids with finite dimensions. The artificial-wall effect created by these grid far-field boundaries must therefore be minimized so as not to degrade the resulting numerical solution.

Various approaches have been developed 10 reduce this spurious influence of far-field boundaries on interior solutions, some of which are listed below:

1) It is common' to place these boundaries far enough (iypically hundreds of chord lengths) from the airfoil such that reflected waves have been greatly reduced in amplitudes by numerical dissipation through the travel from these boundaries. Such a procedure naturally requires a large number of grid points.

2) In a series of papers, Engquist and Majda ${ }^{2-4}$ developed a nonreflecting boundary condition procedure. By absorbing waves incident on the boundary in certain directions, they designed approximate local conditions for simulating the farfield effects along those boundaries. Their lowest order approximation can be interpreted as a simplified form of the characteristic equation.

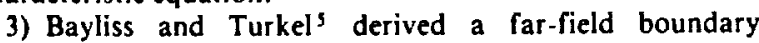
condition procedure based on an asymptotic expansion to any order in the reciprocal of the distance from the origin. The dependence of the far-field distance is shown in the work of Fung, ${ }^{6}$ where the linearized unsteady small-disturbance transonic flow equation is solved to get a far-field condition. This condition is equivalent to a vortex solution with a time lag for waves originating at the airfoil and propagating to the far-field boundary.

4) A viscous damping method which absorbs incident waves on the far-field boundaries was tried by Bushby and

\footnotetext{
Presented as Paper 80-1393 at the AIAA 13th Fluid and Plasma Dynamics Conference, Snowmass, Colo., July 14-16, 1980; submitted Sept. 18, 1980; revision received June 8,1981 . This paper is declared a work of the U.S. Government and therefore is in the public domain.

- Research Scientist, Applied Computational Aerodynamics Branch.
}

Timpson. ${ }^{7}$ This method, however, requires a considerable number of grid points near the far-field boundary.

5) Guderley ${ }^{8}$ (see also Krupp and Cole ${ }^{9}$ ) has shown that in a limited number of cases, far-field conditions can be derived analytically to investigate basic mathematical concepts. However, an extension of this to more general nonlinear problems is not clear at this point.

Unsteady transonic now regions are encountered in the flight of many existing flight vehicles. Typical examples include a fluttering airfoil and spinning helicopter rotors. ${ }^{10}$ For unsteady small-disturbance transonic flows, Ballhaus and Goorjian' have developed a time-accurate implicit finitedifference computer code, LTRAN2. Their boundary conditions at the top, bottom, and upstream boundaries (see Fig. 1) are perfectly reflecting conditions and are the proper ones for boundaries infinitely far away. In the present work, approximate nonreflecting boundary conditions are employed in this code to bring the far-field boundaries closer to the airfoil. As illustrated in Fig. 1, waves originating from the airfoil propagate to the far-field boundaries. The old boundary which is placed at a large distance from the airfoil leaves the near-field solution unaffected by reflected waves from the outer boundary. With nonreflecting conditions, the new boundary can be located closer to the airfoil, thus reducing the area of computation and thereby increasing the degree of resolution within the computational domain.

Among the approaches listed above, the approximate local condition procedure designed by Engquist and Majda ${ }^{24}$ was selected to be used in the present study. Their conditions are simple to implement and require only a modest change in the existing computer code; however, these conditions result in a marked improvement in the computational efficiency of that code.

In Sec. Il of this paper, the governing equation and the boundary condition procedure by Engquist and Majda are presented. In Sec. III, the finite-difference scheme for implementing these conditions in LTRAN2 is described. In Sec. IV, computed results using the old reflecting far-field boundary conditions and those using the new nonreflecting conditions are compared with large grid solutions.

\section{Governing Equation and Far-Field Boundary Conditions}

\section{A. Governing Equation}

The unsteady, transonic small-disturbance equation for low reduced frequencies can be written as

$$
A \phi_{x t}=B \phi_{x x}+\phi_{y y}
$$




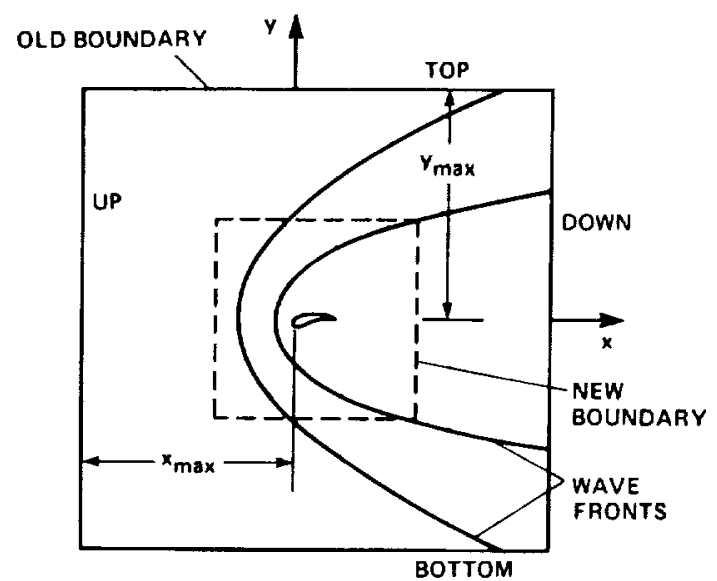

Fig. 1 Schematic of computalional domain: old reflecting boundary vs new nonreflecting boundary.

where

$$
\begin{aligned}
& A=2 k M_{\infty}^{2} / \delta^{2 / 3} \\
& B=\left(I-M_{\infty}^{2}\right) / \delta^{2 / 3}-(\gamma+I) M_{\infty}^{m} \phi_{x}
\end{aligned}
$$

Here, $\phi$ is the disturbance velocity potential, $\delta$ the airfoil thickness-to-chord ratio, and $M_{\infty}$ the freestream Mach number. The reduced frequency is defined as $k=\omega c / U_{\infty}$ for an airfoil of chord length $c$ executing some unsteady oscillatory motion of frequency $\omega$. The choice of the exponent $m$ is somewhat arbitrary and is made to extend the Mach number range of the small-disturbance theory (a rather complete review is given in Ref. 10). The quantities $x, y, t$, and $\phi$ have been scaled by $c, c / \delta^{1 / 3}, \omega^{-1}$, and $c \delta^{2 / 3} U_{\infty}$, respectively. In deriving Eq. (la), it is assumed that

$$
k-\delta^{2 / 3}-\left(1-M_{\infty}^{2}\right)<1
$$

\section{B. Far-Field Boundary Conditions}

In LTRAN2, Eq. (la) is solved for the flow about airfoils by an alternating-direction implicit (ADI) finite-difference algorithm. The existing far-field boundary conditions imposed in LTRAN2 are

$$
\begin{array}{lrl}
\text { upstream: } & \phi=0 \\
\text { downstream: } & \phi_{x}=0 \\
\text { top and bottom: } & \phi_{y}=0
\end{array}
$$

These conditions are perfectly reflecting. In other words, all of the waves originating from the airfoil for unsteady problems are reflected back into the computational domain from the boundaries. Hence, the outer boundaries must be placed far enough from the airfoil in the original version of LTRAN2.

Following Engquist and Majda, ${ }^{4}$ conditions are introduced to absorb at least a portion of the waves incident on the outer boundaries. For the upstream condition, $B$ in $\mathrm{Eq}$. (lb) is assumed to be locally constant; therefore, the analysis is performed on a linear equation. This approximation assumes that the flowfield is governed by a linear equation in the vicinity of a far-field boundary. By considering waves traveling left from the interior to the upstream boundary for subsonic freestream, a perfectly nonreflecting condition is derived. Since this condition requires information from the mathematical domain of dependence of that boundary, approximations are made to get the following local conditions

upstream $\begin{cases}\text { 1st approx: } & \phi_{1}-\frac{B}{A} \phi_{x}=0 \\ \text { 2nd approx: } & \phi_{x t}-\frac{A}{B} \phi_{n}+\frac{l}{A} \phi_{y y}=0\end{cases}$

The first approximate condition absorbs waves normally incident on the boundary, while higher approximations absorb portions of obliquely incident waves.

Since waves travel infinitely fast in the downstream direction, the following consistency conditions seem appropriate (see Krupp and Cole ${ }^{9}$ )

$$
\text { downstream }\left\{\begin{array}{l}
\phi_{x}=0 \\
\phi_{x x}=0
\end{array}\right.
$$

Considering waves traveling to the top and bottom boundaries, a nonreflecting condition is developed. Sub. sequently, local approximations to it are made, resulting in

$$
\begin{aligned}
& \text { top and } \\
& \text { bottom }
\end{aligned}\left\{\begin{array}{l}
\text { 1st approx: } \phi_{y} \pm \sqrt{|B|} \phi_{x}=0 \\
\text { 2nd approx: } \phi_{y y}+r_{2} \phi_{x y} \pm r_{l} \phi_{x l}=0
\end{array}\right.
$$

Here, \pm corresponds to top and bottom boundaries, and $r_{1}$, $r_{2}$ govern the absorption of waves obliquely incident to the boundaries. The values $r_{1}, r_{2}$ are discussed in more detail by Engquist and Majda. ${ }^{4}$

Alternatively, a nonreflecting far-field condition can be designed by considering characteristic equations. Assuming $B=$ const in Eq. (lb) at a large distance from the airfoil, the characteristic equation can be written as

$$
B \xi_{x} \xi_{x}+\xi_{y} \xi_{y}-A \xi_{x} \xi_{1}=0
$$

This equation is satisfied by the characteristic plane

$$
\xi(x, y, t)=r-(x / \sqrt{B}+2 \sqrt{B} t / A)=\text { const }
$$

where

and

$$
\begin{aligned}
& r=(x / \sqrt{B}, y) \\
& \phi=f(\xi)
\end{aligned}
$$

is a solution to Eq. (1a) which represents a traveling plane wave. From these, the following equation is derived by forcing linear combinations of the derivatives of $\phi$ to be zero.

$$
\frac{x}{r} \phi_{x}+\frac{y}{r} \phi_{y}+\frac{l}{2}\left(-\frac{A}{B} \frac{x}{r}+\frac{A}{\sqrt{B}}\right) \phi_{t}=0
$$

This equation can be used as a nonreflecting far-field boundary condition. Since this equation requires the evaluation of $x / r$ and $y / r$ along the boundary at each time step, a further simplification is made. In the upstream region, $x \rightarrow-\infty$ with $|y|$ finite, so that $\mathrm{Eq}$. (12a) becomes

$$
\phi_{1}-(B / A) \phi_{x}=0
$$

For the top and bottom boundaries, $y \rightarrow \pm \infty$ with $|x|$ finite, so that Eq. (12a) becomes

$$
\phi_{y} \pm 1 / 2(A / \sqrt{B}) \phi_{1}=0
$$

Replacing $\phi$, by $\phi_{r}$ using Eq. (IIb), this equation can be written equivalently as

$$
\phi_{y} \pm \sqrt{B} \phi_{x}=0
$$


These are the first approximate relations obtained by Engquist and Majda. Therefore, we may interpret Eqs. (4) and (8) as special forms of a far-field characteristic relation designed for simple, yet very efficient, applications.

\section{Finite-Difference Scheme}

In LTRAN2, ${ }^{1} \mathrm{Eq}$. (1a) is solved by a two-step procedure to advance from time step $n$ to $n+1$

$$
\begin{aligned}
& x \text { sweep: } A(\Delta l)^{-I} \delta_{x}\left(\bar{\phi}_{j, \ell}^{n+l}-\phi_{j, l}^{n}\right)=D_{x} f_{j, \ell}+\delta_{y y} \phi_{j, \ell}^{n} \\
& y \text { sweep: } A(\Delta l)^{-1} \delta_{x}\left(\phi_{j, \ell}^{n+l}-\bar{\phi}_{j, l}^{n+l}\right)=1 / 2 \delta_{y y}\left(\phi_{j, l}^{n+I}-\phi_{j, l}^{n}\right)
\end{aligned}
$$

The operators in Eqs. (13) and (14) are defined by the following equations

$$
\begin{aligned}
& \delta_{x} \phi_{j, p}=\underset{\text { (first order) }}{2\left(\phi_{j, \ell}-\phi_{j-1, \ell}\right)\left(x_{j+1}-x_{j-1}\right)^{-1}} \\
& =\left(3 \phi_{j, 1}-4 \phi_{j-1, t}+\phi_{j-2, t}\right)\left(x_{j+1}-\dot{x}_{j-1}\right)^{-1} \\
& \delta_{y,} \phi_{j, \ell}=2\left[\left(\phi_{j, \ell+1}-\phi_{j, \ell}\right)\left(y_{\ell+1}-y_{\ell}\right)^{-1}\right. \\
& \left.-\left(\phi_{j, t}-\phi_{j, t-1}\right)\left(y_{t}-y_{t-1}\right)^{-1}\right]\left(y_{t+1}-y_{t-1}\right)^{-1} \\
& f_{j, l}=1 / 2\left[B_{j, 1}^{n} \phi_{x_{j, 1}}^{n+1}+\left(1-M_{\infty}^{2}\right) \phi_{x_{j, \ell}}^{n} / \delta^{2 / 3}\right] \\
& B_{j, 1}^{n}=\left(1-M_{\infty}^{2}\right) / \delta^{2 / 3}-(\gamma+1) M_{\infty}^{m} \phi_{x_{j, l}}^{n} \\
& \phi_{x_{j+1 / 2,1}}=\left(\phi_{j+1, f}-\phi_{j, f}\right)\left(x_{j+1}-x_{j}\right)^{-1} \\
& D_{x} f_{j . t}=2\left(x_{j+1}-x_{j-1}\right)^{-1}\left[\left(1-\epsilon_{j}\right)\left(f_{j+1 / 2,1}-f_{j-1 / 2,1}\right)\right. \\
& \left.+\epsilon_{j-1}\left(f_{j-1 / 2,1}-f_{j-3 / 2, t}\right)\right] \\
& \epsilon=\mid l_{1}^{0} \text { for }\left(B_{j+1 / 2,1}^{n}+B_{j-1 / 2,1}^{n}\right) \mid \geqslant 10
\end{aligned}
$$

Here, $\Delta t$ is the time step and $j, \ell$ are the grid point indices in the $x$ and $y$ directions.

The first approximate nonreflecting far-field boundary conditions are readily incorporated into the above twodimensional sweep procedure. For the $x$ sweep, the upstream boundary condition [Eq. (12b)] becomes

$$
\begin{aligned}
& (\Delta t)^{-1}\left(\bar{\phi}_{j+1 / 2, f}^{n+1}-\phi_{j+1 / 2, t}^{n}\right)-1 / 2 B_{j+J / 2, l^{-1}}(\Delta x)^{-1} \\
& \times\left[\left(\bar{\phi}_{j+1.1}^{n+1}-\bar{\phi}_{j .1}^{n+1}\right)+\left(\phi_{j+1.1}^{n}-\phi_{j .1}^{n}\right)\right]=0 \\
& j=1, \mathrm{f}=1, \ldots, \mathrm{fmax}
\end{aligned}
$$

Here

$$
\begin{aligned}
& B_{j+1 / 2,1}=\left[\left(I-M_{\infty}^{2}\right) / \delta^{2 / 3}\right]-\left[(\gamma+I) M_{\infty}^{m}\left(\phi_{j+1,1}^{n}-\phi_{j, l}^{n}\right)\right. \\
& \left.\quad \times\left(x_{j+1}-x_{j}\right)^{-l}\right], \quad j=I
\end{aligned}
$$

The downstream boundary condition is

$$
\bar{\phi}_{j \max , t}^{n+1}+\phi_{\text {max }, t}^{n}-\bar{\phi}_{j \max }^{n+1}-1 . f-\phi_{j \max -1, t}^{n}=0
$$

or equivalently, since $\phi_{j \max , f}^{n}-\phi_{j \max -1, f}^{n}=0$ from the previous iteration

$$
\bar{\phi}_{j \max , t}^{n+l}=\bar{\phi}_{j \max -l, t,}^{n+l} \ell=I, \ldots, \max
$$

Here, jmax and $\max$ are maximum indices in $x$ and $y$ directions and represent downstream and top boundaries, respectively.
Similarly, for the $y$ sweep, the top and bottom boundary conditions become

$$
\begin{aligned}
& \left(\phi_{j-}^{n+1 / 2,1}-\phi_{j-1 / 2, t-1}^{n+1 / 2}\right) /\left(y_{1}-y_{1-1}\right) \pm\left|B_{j-1 / 2,1-1 / 2}\right|^{1 /} \\
& \quad \times\left(\phi_{j, t-1 / 2}^{n+1 / 2}-\phi_{j-1,1-1 / 2}^{n+1 / 2}\right) /\left(x_{j}-x_{j-1}\right)=0 \\
& j=2, \ldots, j \max
\end{aligned}
$$

where

$$
\begin{aligned}
& \phi_{j \pm l / 2 . t}=1 / 2\left(\phi_{j . t}+\phi_{j \pm l . \ell}\right) \\
& \phi^{n+J / 2}=1 / 2\left(\phi^{n+1}+\phi^{n}\right)
\end{aligned}
$$

When $j=2$, Eq. (17) requires $\phi$ at $j=1$. Therefore, for $j=2$ we use Eq. (12b) also. Here, the upstream condition Eq. (15) is changed to

$$
\begin{aligned}
& (\Delta t)^{-1}\left(\phi_{j+1 / 2, \ell}^{n+l}-\phi_{j+1 / 2, l}^{n}\right)-1 / 2 B_{j+1 / 2, e^{-1}}(\Delta x)^{-1} \\
& \times\left[\left(\phi_{j+l, \ell}^{n+l}-\phi_{j, \ell}^{n+l}\right)+\left(\phi_{j+1, \ell^{n}}^{n}-\phi_{j, l}^{n}\right)\right\}=0 \\
& j=l, \ell=I, \text { or } \mathrm{P} \max
\end{aligned}
$$

Then Eqs. (17) and (18) are solved simultaneously.

The higher-order approximations can be applied in a similar way, however, at the expense of increased computing time.

\section{Computed Results for Various Test Cases}

In the production version of LTRAN2, the default grid boundaries are located 857 chords from the airfoil in $y$ direction and 200 chords from the leading edge, with smoothly stretched grid spacings in both directions (113, 97 mesh in $x, y$ directions). The large grid solutions in Figs. 2-7 are obtained using this grid. However, depending on the particular problem, equivalent results can be obtained with smaller grids, and the minimum number of mesh points required is shown in the figures. Therefore, identical solutions are obtained when far-field boundaries are placed farther away than the minimum required.

In computing the large grid solutions, two different sets of far-field boundary conditions are imposed independently, namely: 1) perfectly reflecting conditions as given by Eq. (3); and 2) the first approximate conditions as given by Eqs. (4), (6), and (8). These boundary conditions yield essentially identical results, i.e., within plottable accuracy. Since the large grid results can be duplicated using different combinations of grids and boundary conditions, it is reasonable to assume that these are solutions of $\mathrm{Eq}$. (1) with far-field boundaries at infinity.

\section{A. Step Change in Angle of Attack}

An impulsively started airfoil in plunging motion creates a pressure pulse. This is simulated numerically by a step change in the angle of attack. Due to the low-frequency nature of the governing equation, the lift and moment coefficients gradually increase after the initial change in the angle of attack. A problem such as this provides a good test case for investigating the influence of the computational far-field distances on the flow solution near the airfoil.

Starting from the standard large grid, the far-field boundary distances are reduced by removing outer grid lines in the $x$ (both upstream and downstream equally) and $y$ directions. Both the old reflecting boundary conditions [Eq. (3)] and the new nonreflecting ones are tested with these reduced grids. In Figs. 2 and 3, the lift and moment coefficients for an NACA 64 A006 airfoil are plotted on a time scale in units of chord lengths of airfoil traveled. The indicial responses to a unit change in the angle of attack $\alpha$ are $C_{b_{\alpha}}$ and $C_{m_{\alpha}}$, as described in Ref. 11, and can be regarded as 


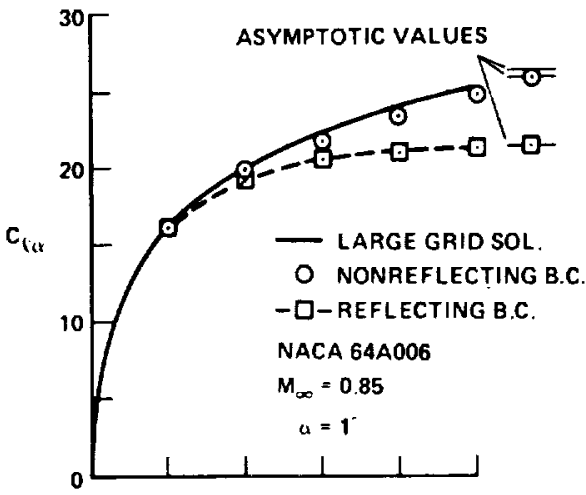

a) LIFT COEFFICIENT

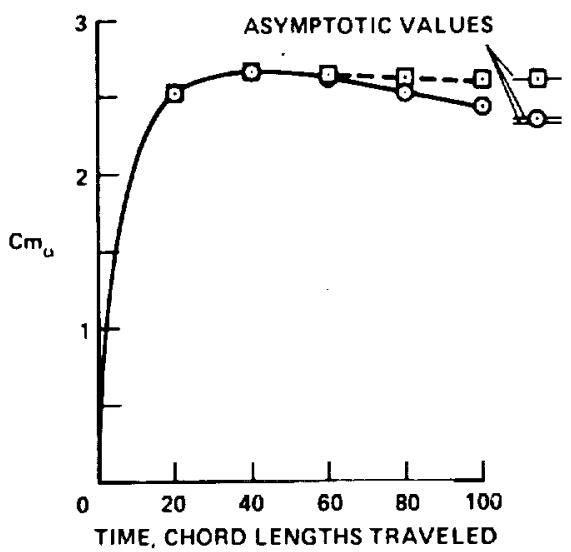

b) MIDCHORD PITCHING MOMENT COEFFICIENT

Fig. 2 Elfect of far-field boundary dislance on $C_{l_{\alpha}}$ and $C_{m_{\alpha}}$ vs $t$ for step change in angle of altack.

$C_{p} / \alpha$ and $C_{m} / \alpha$ in this case. By using the old conditions, the computed results start to deviate from the large grid solutions when the mesh gets smaller than $103 \times 81$ at $M_{\infty}=0.80$ and 0.85 . However, applying the first approximate nonreflecting conditions, the mesh can be reduced to $93 \times 71$ at $M_{\infty}=0.80$ without significant deviations from the large grid results. Similar results are obtained at $M_{\infty}=0.85$, as shown in Fig. 2 .

Since the $x$ boundary can be brought to 3.8 chords from the leading edge úsing the first approximate condition, the second approximation was tried to bring the $y$ boundary closer. However, there was practically no improvement to justify the additional computation required to implement the second approximation. In Table 1 , the minimum mesh and the computational efficiency required to use the old and the first approximate boundary conditions are shown. Using the nonreflecting boundary conditions, the computational efficiencies gained are 19 and $10 \%$ at $M_{\infty}=0.80$ and 0.85 , respectively.

\section{B. Oscillating Flap Case}

Another test of the nonreflecting boundary conditions was performed for the case of an oscillating trailing-edge flap. The configuration consisted of an NACA 64 A006 airfoil with a sinusoidally oscillating flap. Of the three types of possible shock-wave motions, type A (sinusoidal shock-wave motion) and type B (interrupted shock-wave motion) are shown in Figs. 4 and 5 . In both cases, large grid results can be obtained with a grid as small as $93 \times 77$. Again the minimum $x$ boundary distance is very small. As expected, the influence of the $y$ boundary is very pronounced. By the time the $y$ boundary distance is reduced to 7.6 chords from the airfoil, the old reflecting conditions cause significant deviation of the lift and the moment coefficient from the large grid solution.
ASYMPTOTIC VALUES

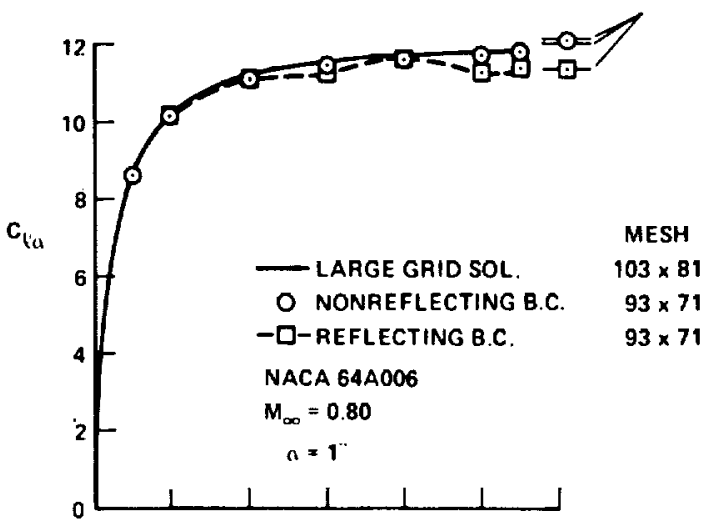

a) LIFT COEFFICIENT

ASYMPTOTIC VALUES

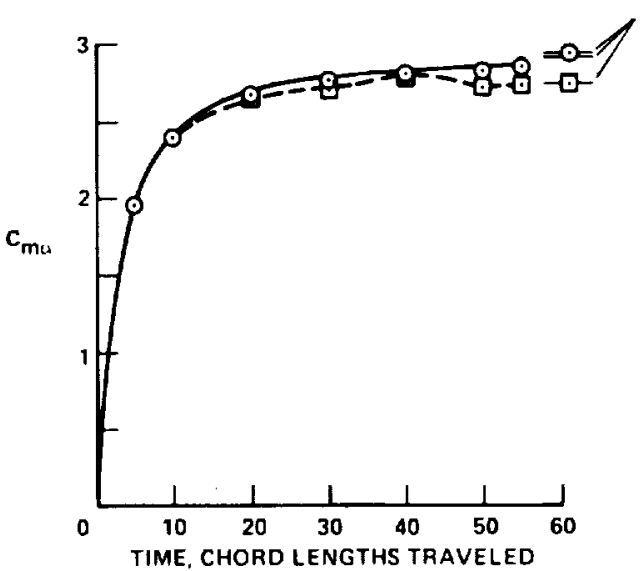

b) MIDCHORD PITCHING MOMENT COEFFICIENT

Fig. 3 Elfect of far-field boundary distance on $C_{t_{\alpha}}$ and $C_{m_{\alpha}}$ for step change in angle of altack.

Table 1 Indicial response to a step change in angle of attack: NACA 64A006, $\alpha=1$ deg $\left(X_{\max }\right.$ and $Y_{\max }$ are given in units of chord)

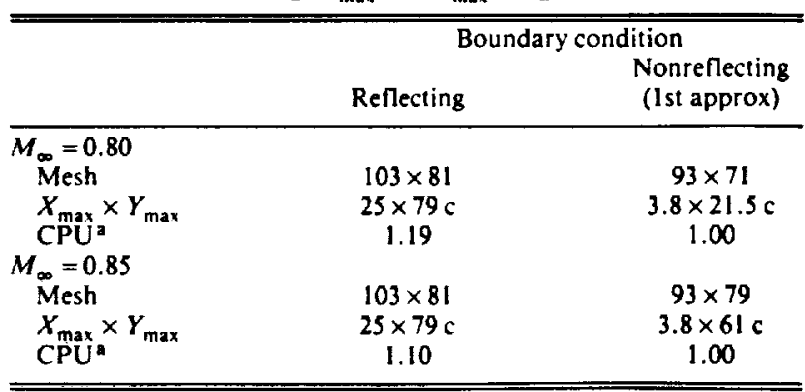

"CPU time is normalized by the nonreflecting case.

Table 2 Sinusoidally oscillating trailing-edge flap: NACA 64A006, $\theta=\sin w$

\begin{tabular}{lcc}
\hline & \multicolumn{2}{c}{$\begin{array}{c}\text { Boundary condition } \\
\text { Nonreflecting } \\
\text { (Ist approx) }\end{array}$} \\
\hline Mesh & $93 \times 77$ & $93 \times 61$ \\
$X_{\max } \times Y_{\max }$ & $3.8 \times 47 \mathrm{c}$ & $3.8 \times 7.6 \mathrm{c}$ \\
$\mathrm{CPU}$ & 1.24 & 1.00 \\
\hline \hline
\end{tabular}

${ }^{3} \mathrm{CPU}$ time is normalized by the nonreflecting case. 
Fig. 4 Efrect of far-field distance on $C_{\text {and }}$ an for an NACA $64 A 006$ airfoil with oscillating Irailing-edge flap: a) type $A$, $M_{-0}=0.875, k=0.468 ;$ b) type B. $M_{\infty}^{\infty}=0.854, k=0.358$.
Fig. 5 Effect of far-field distance on upper surface pressure coerficient for an NACA 64A006 airfoil with oscillating trailing-edge flap: a) lype $A, M_{\infty}=0.875$, $k=0.468 ;$ b) type B, $M_{\infty}=0.854$, $k=0.358$.
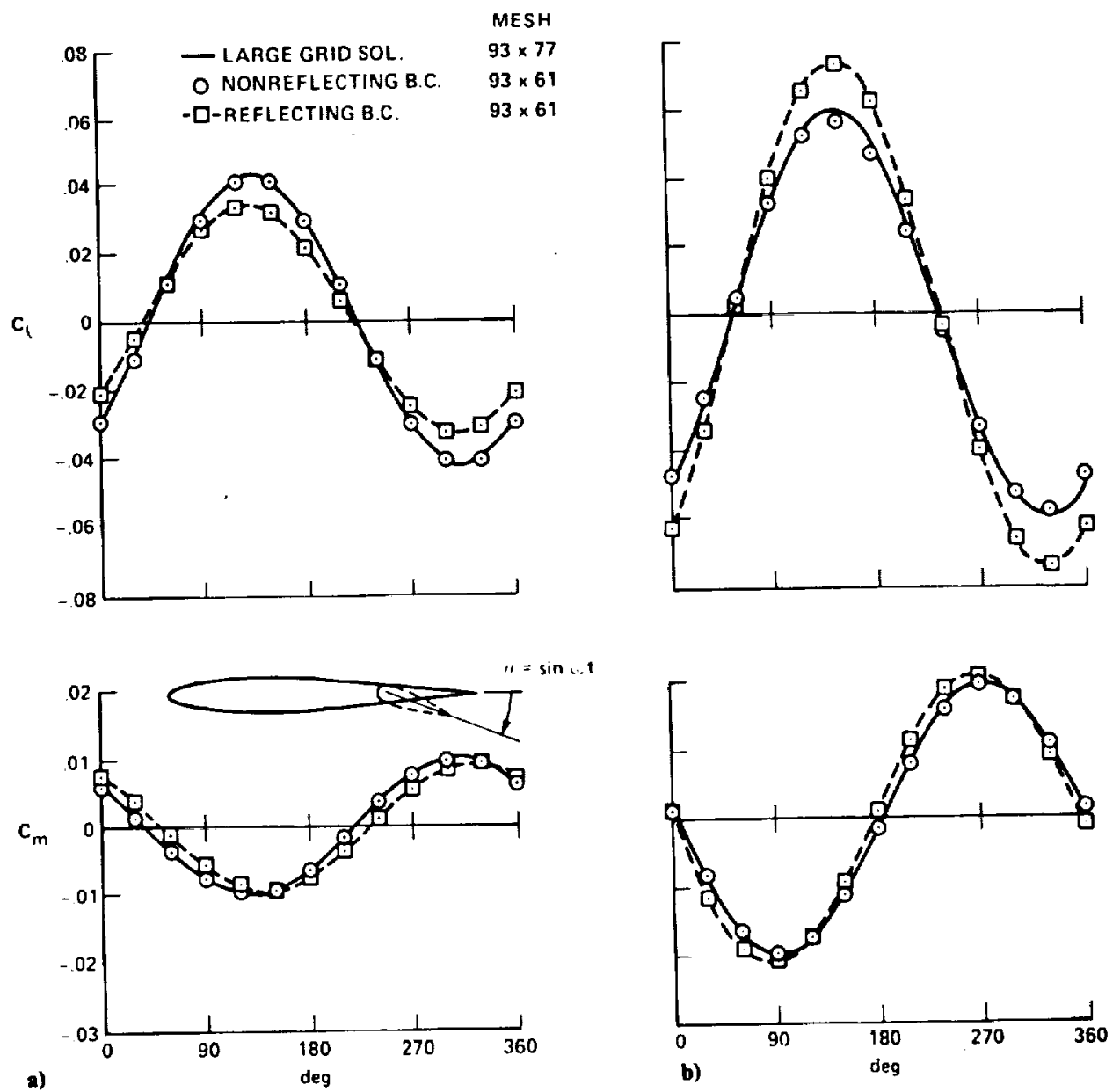

b)

MESH

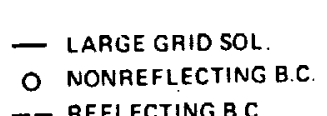

$93 \times 77$

$93 \times 61$ - - REFLECTING B.C

$93 \times 61$
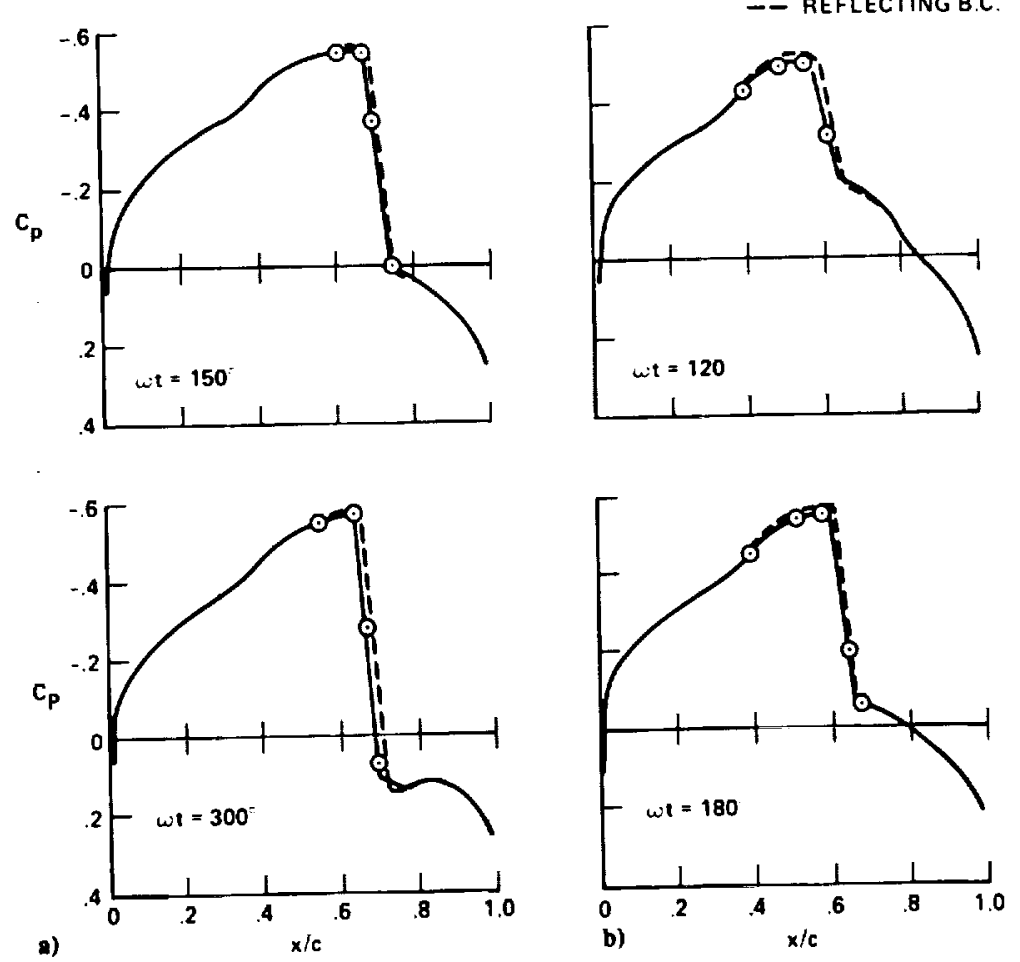
TIME, CHORD LENGTHS TRAVELED

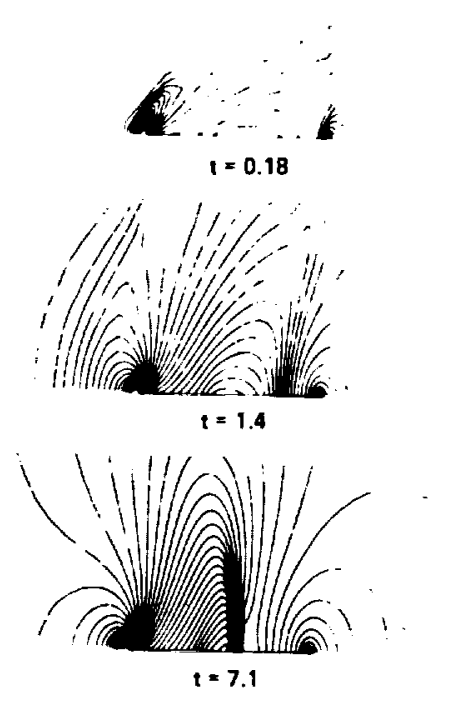

a) LARGE GRID SOLUTION
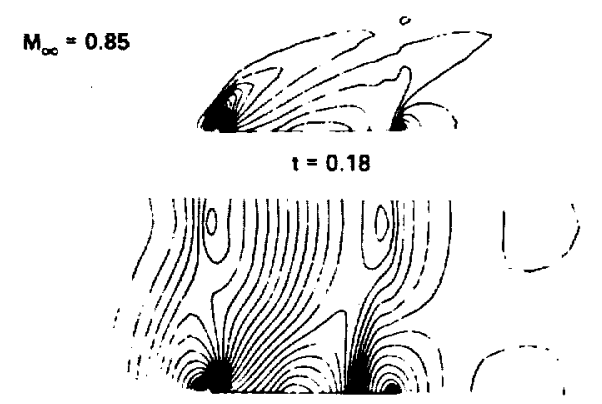

$t=1.4$

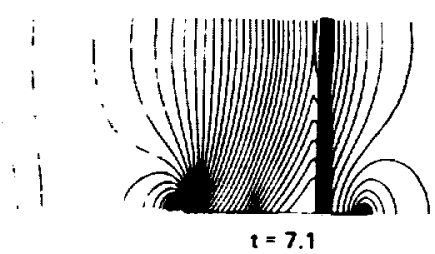

b) REFLECTING B.C.

Fig. 6 Pressure contour for an impulsively starled NACA 64 A006 airfoil from rest.
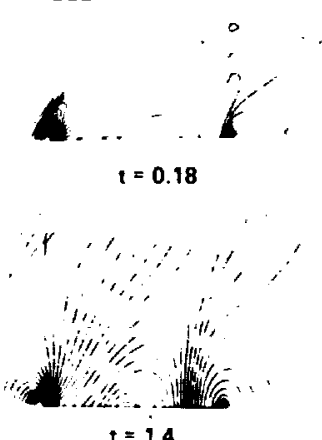

$t=9.4$

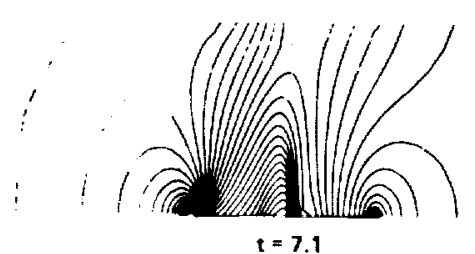

c) NONREFLECTING B. C.
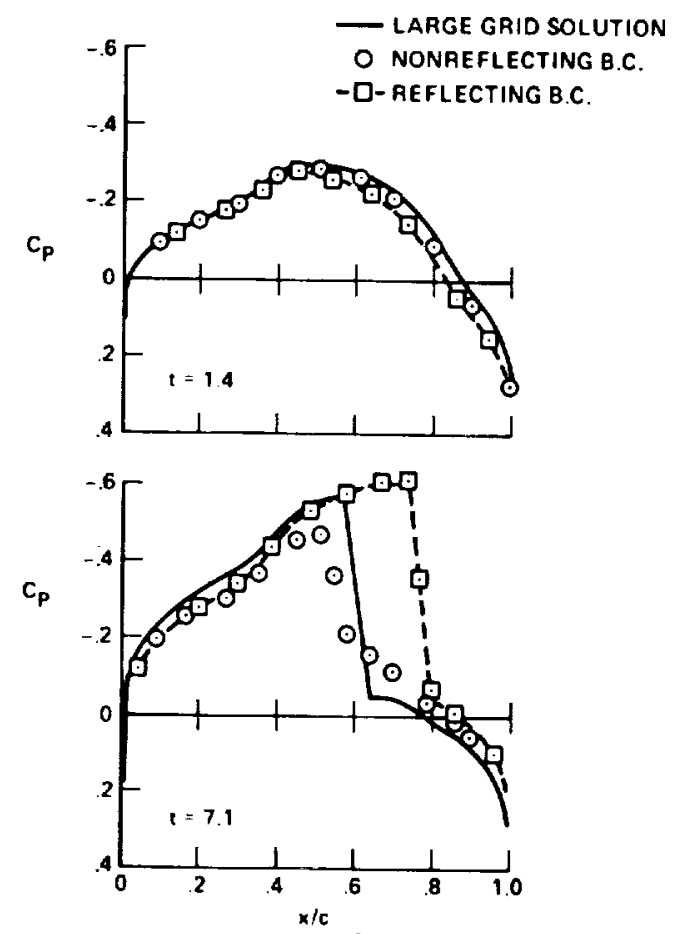

TIME. CHORD LENGTHS TRAVELED

Fig. 7 Upper surface pressure coefficient for an impulsively slarted NACA $64 A 006$ airfoil.

As shown in Fig. 5, when the reflecting boundary is located 100 close to the airfoil, reflected waves cause the shocks to move slightly downstream in both the type $A$ and type $B$ motion. The reflecting boundary conditions on the top and bottom, $\phi_{y}=0$, model solid-wall boundaries. Hence, this close-in imposition restricts the flow and produces stronger shocks. ${ }^{12}$ However, applying nonreflecting conditions, waves incident on the far-field boundaries are mostly absorbed. yielding reasonably good results as shown in Figs. 4 and 5 . It is to be noted that slight changes in $C_{p}$ profile (Fig. 5 ) produce significant changes in unsteady loads (Fig. 4). The gain in computational efficiency, due 10 the first approximate conditions as well as the reduction in computational domain, is shown in Table 2.

\section{Impulsively Started Airfoil}

To visualize the behavior of waves originating from the airfoil and then propagating throughout the computational domain, an NACA 64A006 airfoil is impulsively started from rest at time zero with $M_{\infty}=0.85$. This is equivalently done in LTRAN 2 by turning on the freestream from rest. To see the reflection more clearly, the far-field boundaries are placed very close to the airfoil $\left(X_{\max }=1.22 \mathrm{c}, Y_{\max }=1.08 \mathrm{c}\right)$. The propagation of disturbances is demonstrated by the pressure contour of the upper half plane of the computational domain. In Fig. 6, the results are shown at three sequential times measured by chord lengths of airfoil traveled.

Figure $6 \mathrm{a}$ is obtained from the large grid solution by looking through the window of $1.22 \times 1.08 \mathrm{c}$. In this sequence taken from a movie produced from the calculation, waves from the airfoil propagate outwardly only during the time observed as though the domain were infinite. In Fig. 6b, the old reflecting conditions are used. Physically, this is equivalent to placing solid walls on the boundaries. As time increases, the influence of the boundaries, especially the $y$ boundary, becomes more apparent and, at $t=7.1$, the flow is shown to be choked. This illustrates a possible influence that a solid wind-tunnel wall can have on experimental results. When the nonreflecting conditions are used (Fig. 6c), the pressure contour map more closely resembles the large grid solution. As shown by the upper surface pressure coefficient plot in Fig. 7, the reflecting conditions contaminate the nearfield solution much faster. Since the nonreflecting conditions used are simple approximations to perfectly absorbing conditions, some reflections still exist.

\section{Conclusion}

The first approximate nonreflecting far-field boundary condition procedure of Engquist and Majda has been successfully applied in solving various practical problems using the unsteady small-disturbance transonic flow code, LTRAN2. (As discussed in Sec. IIB, their first approximation is a form of the characteristic equation. Therefore, the boundary condition procedure based on the first approximation alone may be regarded as a characteristic condition procedure.) It has been demonstrated successfully that the computational domain can be reduced considerably using their boundary condition procedure. However, since the mesh system is stretched in LTRAN2, the number of computational grid points is not linearly proportional to the distance of the boundary from the airfoil. The gain in computer time after 
implementing the new conditions is problem dependent and, for the cases tested using the first approximate conditions, the time saved is $10.24 \%$. Further reduction in the computational mesh may be achieved by higher-order approximations. However, the benefit obtained by the reduced number of mesh is overshadowed by the increased computing time required to implement these high-order boundary conditions. Since the approximate conditions applied here are very simple and do not permit any waves to propagate in from the outside, modifications can be made to accommodate the waves which should come back into the domain. However, especially in the production version of a code, the simplicity of the first approximate nonreflecting conditions and the computational efficiency gained are more than adequate for applications in the design process.

\section{References}

'Ballhaus, W. F. and Goorjian, P. M., "Implicit Finite-Difference Computations of Unsteady Transonic Flows About Airfoils," $A I A A$ Journal, Vol. 15, Dec. 1977, pp. 1728-1735.

'Engquist, B. and Majda, A., "Absorbing Boundary Conditions for the Numerical Simulation of Waves," Mathemarics of Computation, Vol. 31, No. 139, July 1977, pp. 629-651.

${ }^{3}$ Engquist, B. and Majda, A., "Radiation Boundary Conditions for Acoustic and Elastic Wave Calculations," Communications on Pure and Applied Mathematics, Vol. 32, May 1979, pp. 313-357.
4Engquist, B. and Majda, A., "Numerical Radiation Boundary Conditions for Unsteady Transonic Flow," Journal of Compurational Physics, Vol. 40, No. I, March 1981, pp. 91-103.

${ }^{5}$ Bayliss, A. and Turkel, E., "Radiation Boundary Conditions for Wave-like Equations," ICASE Rept. 79-26, NASA Langley Research Center, Oct. 1979.

${ }^{6}$ Fung, K.-Y., "Far-Field Boundary Conditions for Unsteady Transonic Flows," AIAA Journal, Vol. 19, Feb. 1981, pp. 180-183.

'Bushby, F. H. and Timpson, M. S., "A 10-level Atmospheric Model and Frontal Rain," Quarterly Journal of the Royal Meteorological Society, Vol. 93, 1967, pp. I.17.

${ }^{8}$ Guderly, K. G., "Far Field Conditions for Subsonic Flows with Small Superimposed Harmonic Oscillations," AFFDL-TR-79.3109, July 1979.

${ }^{9}$ Krupp, J. A. and Cole, J. D., "Studies in Transonic Flow IV, Unsteady Transonic Flow," UCLA-ENG-76104, Oct. 1976.

${ }^{10}$ Ballhaus, W. F., "Some Recent Progress in Transonic Flow Computations," VKI Leciure Series: Compurational Fluid Dynamics. von Kármán Institute for Fluid Dynamics, Rhode-Si-Genese, Belgium. March, 1976.

I"Ballhaus, W. F. and Goorjian, P. M., "Computation of Unsteady Transonic Flows by the Indicial Method," AIAA Journal, Vol. 16, Feb, 1978, pp. 117-124.

iz Ballhaus, W. F. and Goorjian, P. M., "Efficient Solution of Unsteady Transonic Flows About Airfoils," AGARD Specialists Meeting on Unsteady Airloads in Separated and Transonic Flow, Lisbon, Portugal, April 1977, AGARD Conf. Proc. 226, Paper No. 14. 